\title{
Protée
}

\section{Parler du virtuel. La musique comme cas exemplaire de l’icône}

\section{Jean Fisette}

Volume 26, numéro 3, 1998

Logique de l'icône

URI : https://id.erudit.org/iderudit/030525ar

DOI : https://doi.org/10.7202/030525ar

Aller au sommaire du numéro

Éditeur(s)

Département des arts et lettres - Université du Québec à Chicoutimi

ISSN

0300-3523 (imprimé)

1708-2307 (numérique)

Découvrir la revue

Citer cet article

Fisette, J. (1998). Parler du virtuel. La musique comme cas exemplaire de l’icône. Protée, 26(3), 45-54. https://doi.org/10.7202/030525ar

\section{Résumé de l'article}

L'auteur cherche à reproduire les diverses circonstances suivant lesquelles il serait possible de saisir la notion d' ' icône ». En premier lieu, il dresse une liste des principales caractéristiques de l'icône, telles qu'on peut les répertorier dans les textes de Peirce. Puis, une confrontation de ces traits au signe visuel qui devrait, suivant l'étymologie, exemplifier l'icône démontre plutôt que, contre toute attente, cette équivalence entre l'icône et le signe visuel vient créer des difficultés majeures qui sont liées à l'une des questions centrales de toute théorie du signe, à savoir la place que l'on doit réserver à la question de la représentation. Or il s'avère que les caractères reconnus par la majorité des travaux des spécialistes au signe musical correspondraient de façon beaucoup plus juste à l'icône dans la mesure où cette dernière ménage, à l'intérieur du signe, une place à l'imaginaire, d'où il est possible d'appréhender le virtuel et de laisser l'émotion s'inscrire dans le processus de la " sémiosis ". L'auteur évalue cette hypothèse et en mesure les retombées sur la théorie du signe.
Ce document est protégé par la loi sur le droit d'auteur. L'utilisation des services d’Érudit (y compris la reproduction) est assujettie à sa politique d'utilisation que vous pouvez consulter en ligne.

https://apropos.erudit.org/fr/usagers/politique-dutilisation/ 


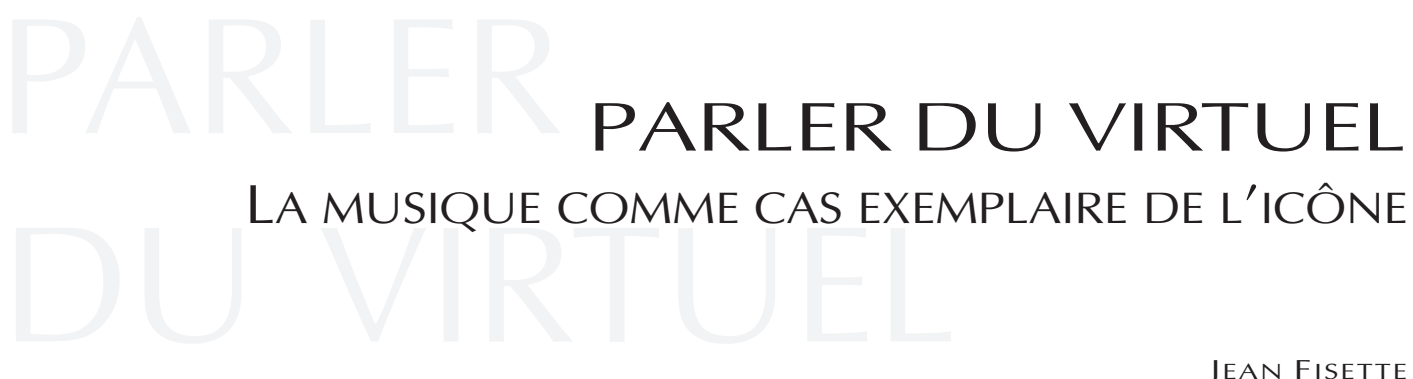

Les sons, dans la mélodie, n'agissent pas seulement sur nous comme sons, mais comme signes de nos affections, de nos sentiments; c'est ainsi qu'ils excitent en nous les mouvements qu'ils expriment, et dont nous y reconnaissons l'image. (J.J. Rousseau, 1781: 84) [...] la musique est émouvante parce qu'elle est mouvante; c'est cette analogie qui nous fait appeler cet art imitateur lorsqu'à peine, il imite. (P. Guy de Chabanon, 1785: 19)

Music is revealing, where words are obscuring because it can have not only a content, but a transient play of contents.

(S. K. Langer, 1942: 243)

Je tenterai de reproduire les diverses circonstances suivant lesquelles nous pouvons arriver à saisir la notion d'icône. Je serai donc appelé, en premier lieu, à dresser, pour mémoire, une liste des principales caractéristiques de l'icône telles que l'on peut les répertorier dans les textes de Peirce. Puis je confronterai ces traits retenus au signe visuel qui devrait, suivant l'étymologie, exemplifier l'icône alors que, contre toute attente, cette équivalence entre l'icône et le signe visuel vient créer des difficultés majeures, qui sont liées à l'une des questions centrales dans toute théorie du signe, à savoir la place que l'on doit réserver à la fonction de représentation. Or il s'avère que les caractères reconnus par la majorité des travaux des spécialistes au signe musical correspondent de façon beaucoup plus juste à l'icône dans la mesure où cette dernière ménage, à l'intérieur du signe, une place à l'imaginaire, d'où il est possible d'appréhender le virtuel et de laisser l'émotion trouver à s'inscrire dans le processus de la semiosis.

Claude Lévi-Strauss (1962: 5) écrivait que, lorsqu'on tente d'analyser, par le biais d'un vocabulaire scientifique, des phénomènes comme l'hystérie ou le totémisme, ils s'évanouissent comme par enchantement, de sorte qu'il devient impossible de les constituer comme notions et donc d'en faire des objets d'étude. Avec la question du virtuel, on rencontre une situation semblable et c'est la raison pour laquelle je m'entoure de précautions lexicales telles: reproduire des circonstances, appréhender le virtuel, imaginer l'icône; de plus, en fin de parcours, je déborderai la 
logique de la table raisonnée des notions et de traits construits avec l'objectif de définir l'icône, pour me référer, plus directement, à l'expérience de l'écoute musicale. Ce ne sont pas là de simples effets de rhétorique, mais des détours auxquels on doit se soumettre pour ne pas chosifier ou ne pas objectiver le virtuel qui, autrement, à la façon de l'hystérie et du totémisme, nous échapperait sûrement.

\section{LE VIRTUEL, L'ICÔNE \\ ET DIVERS TYPES DE REPRÉSENTATION}

L'icône, telle que la définissait Peirce, désigne le premier niveau dans la relation du signe à l'objet, les deux autres étant, dans leur ordonnancement, l'indice et le symbole. Ces trois termes ne constituent pas chez Peirce un paradigme au sens saussurien, mais bien une triade - sans doute la plus connue - et, par conséquent, ils sont à penser sur la base de l'articulation entre les trois catégories de la phanéroscopie que sont la priméité, la secondéité et la tercéité.

Ces quelques éléments du premier tableau de la semeiotic sont suffisamment connus pour qu'il ne soit pas nécessaire d'y revenir. Par contre, on ne saurait se contenter d'une schématisation formelle aussi abstraite, pour la simple raison qu'en cet état elle est pure nomenclature; par ailleurs, lorsqu'on suit les nuances du texte de Peirce, allant au delà de ces découpages élémentaires, on découvre des incertitudes et des hésitations qui ménagent une place à l'imprévu et ouvrent la voie à de nouvelles découvertes.

C'est sur un passage du texte de Peirce, centré sur la notion d'icône, que je construirai ma réflexion:

L'empreinte d'un pied que Robinson Crusoé a découverte dans le sable et qui a été gravée dans le granit de la renommée, était pour lui un indice à l'effet qu'une certaine créature habitait son

île, et, simultanément, en tant que symbole, ce signe appela l'idée d'un homme. Chaque icône partage, de façon plus ou moins évidente, les caractères de son objet. Les icônes partagent tous les caractères les plus évidents des mensonges et des déceptions elles les affichent. [...] L'icône ne se substitue pas de façon univoque à telle ou telle chose existante comme le fait l'indice. Son objet [à l'icône] peut être une pure fiction qui fonde ainsi son existence. Rarement son objet est une chose d'une sorte qu'on rencontre habituellement.

(C. S. Peirce, C.P. 4.531, trad. Fisette, 1996: 276-277. Je compléterai la citation de ce fragment dans la seconde partie de cet article.)

L'empreinte du pied dans le sable est donnée comme un indice permettant d'inférer le passage d'une certaine créature et puis, cumulativement, cette association «empreinte - créature" prend à son tour valeur de symbole, ce qui implique un déplacement du lieu de surgissement du signe, depuis la plage de sable sur l'île jusqu'à l'auteur Daniel Defoe qui, lui, se situe pleinement dans le symbolique, puisqu'il a porté son personnage au "granit de la renommée». Mais alors où placer l'icône dans tout ceci? Le terme icône, dans la mesure où il est très proche du premier constituant du signe (fondement ou representamen), désignerait les contours de l'empreinte, ses effets de profondeur marquant en creux une troisième dimension et, éventuellement, la texture de la matrice imprimée dans le sable. Au sens de Peirce, le terme icône, qui renvoie au matériau fondant la représentation, est très proche de l'artefact. On pourrait alors suggérer que l'icône désigne ce qui, fondant l'échange entre les deux personnages, inscrit une possibilité de sens; pour cette raison, l'icône est la condition première du signe.

\section{L'ICÔNE INSCRIT DANS LE SIGNE}

LA POSSIBILITÉ DE L'IMAGINAIRE

L'icône, a-t-on dit, désigne la relation du signe à l'objet, cette relation étant pensée suivant la logique de la priméité. Et lorsqu'on se réfère à la priméité, on se situe dans une antériorité logique par rapport à l'existence.

La difficulté centrale que rencontrent tous les chercheurs qui tentent de comprendre le virtuel - et les professeurs de sémiotique qui tentent d'expliquer, devant un groupe d'étudiants, la notion d'icône tient à un quasi-paradoxe: aussitôt que l'on arrive à saisir les éléments de contenu correspondant à la priméité, ils sont passés, par cet acte même de saisie, à l'existence et donc à la secondéité. En somme le 
virtuel serait, par définition, insaisissable: en parler autrement que d'une façon circonstancielle relèveraitil d'une impossibilité logique ou d'un paradoxe? Ce n'est pas si sûr! En fait, toute la question est là.

C'est donc dire que pour désigner l'empreinte dans le sable comme une icône, il faudrait se placer dans un très bref laps de temps, dans une durée que l'on imagine plus que l'on ne la saisit réellement, et que l'on pourrait délimiter entre les deux bornes que seraient, pour commencer, le moment où l'image de l'empreinte a atteint le centre de l'intelligence (certains diront le centre de l'«appareil cognitif») et, pour terminer, cet autre moment, où une relation est établie entre l'empreinte et un objet existant à l'extérieur de celle-ci, à savoir, ici, un pied ayant agi comme matrice.

Cet exemple conduit à établir la distinction entre l'existence factuelle et ce qui la précède logiquement et qu'on pourrait appeler le possible; et ce "possible», lorsqu'il est considéré pour lui-même, ne peut être pensé que comme virtualité; ce qui nous conduit à cette étonnante suggestion de Nicole Paquin (1996: 82): «Les décalages entre le perçu et le réel objectif sont à la base même de l'imaginaire»; et effectivement, dans la mesure où la notion d'icône inscrit, à l'intérieur du signe, une place au virtuel, à ce qui n'est pas encore réalisé mais qui est attendu, elle ménage une place à l'imaginaire.

\section{Des Plages DE SABLE AUX RIVES DU RÊVE}

Maintenant, comment définir le virtuel d'un point de vue spécifiquement peircéen ${ }^{1}$ ? Je précise que je tente ici moins de définir l'icône que de saisir les conditions de son appréhension.

On pourrait trouver une première réponse en retournant à la définition stricte des catégories. La priméité se définit par l'absence de distinction ou de discrimination entre des ordres de choses ou entre les divers matériaux qui y logent, de sorte que le signe et son objet forment une même entité floue. La trace dans le sable, pour revenir à l'exemple précédent, reste une icône aussi longtemps qu'un objet extérieur, ayant agi comme matrice, n'a pas encore été reconnu dans l'empreinte, c'est-à-dire aussi longtemps que ces deux constituants du signe n'ont pas encore été discriminés, puis dissociés.

Peirce donne un exemple assez convaincant de cette situation où le signe et son objet s'engluent l'un dans l'autre, perdant leurs identités propres:

[...] en contemplant un tableau, il y a un moment où nous perdons conscience qu'il n'est pas la chose, la distinction entre le réel et la copie disparaît, et c'est sur le moment un pur rêve [...].

À ce moment nous contemplons une icône.

(C.P. 3.362, trad. Everaert-Desmedt)

C'est la narration du rêve, ce qu'on appelle l'élaboration secondaire ${ }^{2}$, qui aura précisément la fonction de venir conférer un effet de réalité au rêve, c'est-à-dire lui reconnaître une existence en dehors de l'appareil psychique d'où il a surgi et qui l'a porté.

\section{CE N'EST PAS UNE RELATION DE SIMILARITÉ} QUI DÉFINIT L'ICÔNE, MAIS UNE SIMPLE FUSION OU UN ÉTAT DE CHAOS

Lorsqu'on définit la relation à l'objet, au niveau de la secondéité, sur la base d'une rencontre entre deux éléments reconnus dans leur distinction - soit, pour revenir à notre exemple, l'empreinte dans le sable et la créature -, on parle d'une relation de contiguitté.

À partir du moment où la relation appartenant à l'ordre de la secondéité, fondée sur un rapport dyadique entre deux éléments, est définie comme une relation de contiguité, il devient inévitable - en raison d'automatismes linguistiques - que la relation appartenant à la priméité soit, suivant la règle linguistique de la différence, caractérisée par le terme de similarité (et la relation troisième par celui de conventionnalité) ${ }^{3}$. Revenons à Robinson et à ses inférences: l'empreinte serait mise en relation de contiguité avec un pied parce qu'elle en constitue la trace. Ou encore un rêve serait mis en relation avec un objet spécifique parce qu'on y aurait reconnu ce même objet?

Mais nous savons très bien que c'est faux, que ça ne fonctionne pas comme cela, parce qu'alors on ferait semblant de rechercher un objet qui serait déjà connu. 
Ca ne fonctionne pas parce que, dans la priméité authentique, prise stricto sensu, dans le décalage de l'imaginaire comme on a dénommé ce laps de temps, le contour, la profondeur et la texture de l'empreinte d'une part, la matrice de l'autre sont indissociables; l'un ne peut pas être similaire à l'autre puisqu'ils se confondent; entre les deux termes, il n'y a pas une relation qui serait à définir mais, plus simplement et plus radicalement, une indifférenciation, une fusion, bref ce que l'on pourrait appeler un état de chaos.

En fait, la similarité sera reconnue après coup; on pourrait même imaginer qu'elle n'est, la plupart du temps, qu'une résultante de l'établissement de la contiguité; comme si la similarité était la façon dont la priméité était perçue et évaluée d'un point de vue dyadique. On n'a qu'à penser aux analyses de rêves, à l'«Homme aux loups", par exemple, et se demander dans quelles circonstances - ou à quel moment - ses pulsions ont été associées à des loups? On pourrait plus sûrement se référer aux épisodes bien connus de La Recherche dont Genette (1976) a bien démontré l'antécédence de la contiguïté sur la similarité dans le processus même de l'écriture.

\section{SI L'ICÔNE EST UN SIGNE,} DE QUOI DONC EST-ELLE SIGNE?

Alors, si l'icône n'est qu'en relation avec elle-même, indifférenciée de son objet, est-elle signe?

Pour répondre, on n'a qu'à reformuler la question, mais en changeant de point de vue, en inversant l'ordre des termes: que serait l'objet d'un signe qui n'aurait pas d'existence indépendante du representamen qui le porte? Ou encore: peut-on imaginer un signe qui serait impensable sans son objet parce qu'il ne s'en distinguerait pas et que, simultanément, il ne le désignerait pas comme une altérité? Ou encore: que serait un signe qui se désignerait lui-même?

La réponse s'impose alors immédiatement à l'esprit: c'est le cas des objet fictifs? On pourrait revenir aux exemples classiques de la licorne et du centaure; et l'on rappellera que ces objets ont inévitablement des connotations sexuelles, le lieu logique de l'icône ayant été illustré par Peirce - on y a fait allusion plus haut - par le recours au rêve, une instance virtuelle donc qui, précédant l'existence et n'étant pas soumise à l'épreuve de la réalité, laisse libre cours à toutes les manifestations de l'imaginaire et du désir.

Mais peut-être, à bien y penser, la licorne et le centaure sont-ils de mauvais exemples, puisqu'ils ont été constitués comme valeurs symboliques depuis belle lurette et qu'ils sont donc reconnus comme tels dans la culture. En deçà des figures constituées, se dressent en fait tous les personnages de fiction qui se construisent dans nos imaginaires, des objets étranges, des lieux de mémoire, toutes matières pré-sémiotiques (dans la logique de Peirce, on les nommerait des «quasi-signes»); ce sont, en fait, des formes-contenus qui, comme un métal liquide, sont encore à l'état de fusion, qui n'ont pas encore acquis droit de cité, qui n'ont pas encore été fixées par la communauté et qui n'ont pas encore été construites comme valeurs.

J'ai déjà suggéré de nommer quasi-symboles ces matières pré-sémiotiques. L'icône constitue le lieu même de génération de la métaphore qui repose précisément sur l'entrechoquement de mots-signes qui, dans ces circonstances, perdent leur frontières sémantiques, leurs discriminations constitutives. Peirce avait d'ailleurs bien vu cela lorsqu'il a proposé l'analyse triadique de l'icône sous la dénomination de l'hypoicône ${ }^{4}$.

\section{L'ICÔNE ET LE SIGNE VISUEL}

Voici un tableau des traits retenus pour appréhender l'icône:

- ordre de la non-réalisation ou ce qui précède logiquement l'existence;

- condition logique de non-discrimination ou d'indifférenciation entre les constituants (logique monadique et non dyadique);

- logique constitutive qui n'est ni une relation de similarité, ni une relation de contiguité mais, plus radicalement, une fusion;

- lieu d'un espace virtuel donné comme une entité floue ou comme un état de chaos;

- lieu de l'imaginaire donné comme un laps de temps, un «décalage entre le perçu et le pensé»; 
- un contenu (inséparable du signe) qui est instable, en gestation constante ou en mouvement;

- l'objet de l'icône ne peut exister qu'au titre d'une fiction qui n'a pas encore été reconnue par la collectivité, et donc non encore construite comme valeur (de l'ordre du symbolique);

- l'icône, dans ces conditions, ressemblerait plutôt à un rêve, c'est-à-dire à une expérience très intime dont, en dehors de l'élaboration secondaire, on n'arrive pas à attester la réalité;

- l'icône est une instance logique que l'on ne saurait saisir sans risquer de la faire disparaître; on ne peut que l'imaginer d'une façon circonstancielle.

Revenons à la question restée en attente depuis le début de cette réflexion: comment imaginer l'icône suivant ces propositions?

La difficulté majeure provient d'habitudes et de formes linguistiques qui, évidemment nécessaires au discours, sont toujours difficilement contrôlables et entraînent des effets de sens plus ou moins désirables.

D'abord, le mot icône: on connaît tous les sens que prend ce mot dans les domaines reliés à l'histoire de l'art et aux sémiotiques visuelles. On sait qu'en retenant le terme «icon», Peirce faisait l'emprunt du mot grec $\varepsilon \imath \kappa \omega v$ [eikône] signifiant simplement «image» (soit l'équivalent du mot latin «imago»; n'oublions pas le contexte de l'imaginaire, évoqué plus haut). Puis, dans la famille du mot grec on trouve, par exemple, le verbe $\varepsilon \imath \kappa \omega$ [eikô] qui signifie «ressembler, paraître». En somme, on aurait toutes les raisons de soupçonner que la difficulté à imaginer l'icône n'est pas étrangère à ce renvoi dominant à la modalité du visible où la notion de ressemblance est centrale.

Car le problème majeur tient à la difficulté, sinon à l'impossibilité pour une image - une représentation visuelle - de répondre à ces traits qui définissent l'icône: car derrière l'image, un objet extérieur est, la plupart du temps, présent ou du moins pressenti; cela suppose la discrimination entre le signe et son objet, donc une relation de contiguïté ou de similarité, puis un certain caractère de stabilité qui est projeté de l'objet extérieur à l'intérieur du signe - ce qui n'est pas le cas avec les objets étranges et les personnages mythiques que nous construisons dans nos rêves collectifs, ces fables que nous inventons et que nous nous racontons. Or, les exemples rencontrés dans le texte de Peirce pour illustrer l'icône renvoient presque exclusivement ${ }^{5}$ à des signes visuels fondés sur de telles relations avec leur objet et qui viennent contredire les caractères de l'icône énumérés plus haut. La difficulté est majeure et elle est liée à un point central resté obscur dans la semeiotic: il y a là comme une tache aveugle dans la construction théorique. C'est que les signes visuels que l'on rencontre dans les Collected Papers sont généralement pensés en fonction de la figuration et, de plus, sont voués à un idéal de représentation.

Or l'icône même, malgré son nom, désigne non pas une représentation, mais une simple présence: Peirce (C.P. 1.313) suggère le terme presentment que Claudine Tiercelin (1993: 158) a traduit par présentité: une simple qualité de présence. L'icône est antérieure à la représentation; elle est plus primaire, car elle ne présuppose rien d'autre qu'elle-même; elle est également logiquement antérieure à la figuration. Ces mêmes raisons expliquent aussi - et c'est significatif que les sémiotiques visuelles aient pu se développer à partir du moment où on s'est donné comme objet d'analyse des images, des dessins, des toiles, etc. qui n'étaient pas soumis à la figuration ou qui ne répondaient pas à une fonction dominante de représentation.

La conséquence de tout ceci: si l'on pouvait généraliser le sens du terme icône pour l'étendre aux modalités sensorielles autres que la vue, le terme trouverait la généralité qui est sienne dans son usage sémiotique et, alors, il gagnerait en extension (et non en compréhension), puis en efficacité et en puissance évocatrice. Je reviens donc aux traits retenus pour définir l'icône et je découvre avec étonnement que si la représentation visuelle me pose à moi des difficultés majeures (qui pourtant ne sont pas essentielles), en revanche, le representamen musical semble venir exemplifier sinon donner du corps à l'ensemble de ces traits ${ }^{6}$; et il le fait, pourrait-on dire, avec une sorte d'évidence. Pour une raison très simple: derrière le 
signe musical, on ne pressent aucunement la menace d'un objet extérieur qui viendrait dyadiser ou référentialiser le signe?

\section{L'ICÔNE MUSICALE}

Pour des raisons d'espace et de concision, je ferai l'économie d'un tableau historique des travaux, par ailleurs extrêmement intéressants, qui ont été conduits dans le cadre de l'esthétique et de la musicologie, pour ne retenir que quelques paragraphes d'un texte qui compte parmi les plus importants qui aient été écrits au XXe siècle sur le statut de la musique en regard des questions centrales de l'imitation, de la capacité représentative (ou de la figuration) et du pouvoir pragmatique du signe musical:

Les lettres algébriques sont de purs symboles; nous voyons des relations algébriques non pas à l'intérieur d'elles mais à travers elles; elles possèdent la plus haute transparence que le langage puisse atteindre. [...] Le message de la musique n'est pas une abstraction, ni un concept fixe comme le serait une leçon de mathématiques. La musique n'est pas transparente mais chatoyante [iridescent]. Ses valeurs se superposent constamment, ses symboles sont inépuisables.

[...] La puissance réelle de la musique tient au fait qu'elle peut être "vraie» de la vie des sentiments d'une façon qui est interdite à la langue; car ses formes signifiantes ont cette ambivalence de contenu inconnue aux mots. La musique révèle alors que les mots obscurcissent parce qu'elle peut avoir non seulement un contenu, mais une réserve de contenus éphémères. La musique peut articuler des sentiments sans se marier à eux. [...] Un simple changement de clef peut entraîner une nouvelle perception sensible du monde (Weltgefühl). L'assignation d'un sens est changeante, un jeu de kaléidoscope, probablement situé sous le seuil de la conscience, certainement en dehors de l'éventail des pensées discursives. [...] Parce qu'aucune détermination de sens n'est conventionnelle, aucune ne dure au delà du son qui passe. Toute brève association n'aura été qu'un éclair de compréhension [a flash of understanding].

[...] L'effet durable ressemble au premier effet $d u$ discours sur le développement de la conscience: celui de rendre les choses concevables plutôt que de les enfermer dans des propositions.
[...] Cela n'a rien à voir avec un traité sur les émotions; c'est beaucoup plus subtil, plus complexe, plus changeant et beaucoup plus important: en somme l'apport total de la musique est celui d'une satisfaction émotionnelle, d'une assurance intellectuelle et d'une compréhension musicale [musical understanding]. La musique a réalisé sa mission lorsque nos cours sont satisfaits.

(Langer, 1942: 239-244. Les caractères en romain sont en italique dans le texte cité; j'ai inscrit entre crochets quelques mots et expressions provenant du texte original; le terme allemand Weltgefühl, placé entre parenthèses, figure dans le texte cité. Trad. Fisette)

Ce texte de Suzanne Langer a paru en 1942. Ces quelques paragraphes en marquent les principales avancées. Toutes les propositions retenues plus haut pour circonscrire l'icône trouvent ici une illustration et une confirmation convaincantes; je fus moi-même extrêmement étonné de cette rencontre et de cet accord $^{8}$. En fait, ce texte reprend l'ensemble des questionnements et des problématiques qui ont été soulevées par la musicologie et l'esthétique musicale et dont la tradition remonte à Rameau, à Rousseau et à Diderot pour finalement proposer une vaste synthèse. Aujourd'hui, cette référence est devenue incontournable pour quiconque s'intéresse aux questions du sens et de la signification en regard du signe musical.

\section{ET LES VALEURS DE VÉRITÉ DE L'ICÔNE?}

Les musicologues n'ont de cesse d'opposer l'impossibilité de validation des effets de sens d'une pièce musicale à leur caractère incontournable, c'est-àdire à la nécessité de reconnaître leur présence fonctionnelle dans toute audition, à défaut de quoi la musique, suivant la position esthétique défendue par Edouard Hanslick (1854), demeurerait une pure construction formelle, étrangère à tout procès de signifiance et à toute forme d'investissement émotif 9 . C'est là présenter en quelques mots un débat central qui anime les discours et les discussions entre musicologues depuis plus de trois siècles et qui pose, avec acuité, une question qui est aussi au cœur de toutes les théories du signe. On pourrait illustrer cette 
question, en regard de la théorie sémiotique de Peirce, en abordant celle de la prédominance entre les divers types de relation du signe à son objet, allant de l'icône au symbole, et aussi entre les diverses classes d'interprétants.

À cet effet, je reviens au fragment textuel qui prolonge le passage cité au début de cette réflexion et qui concerne l'empreinte dans le sable sur l'île de Robinson:

Les icônes partagent tous les caractères les plus évidents des mensonges et des déceptions - elles les affichent. [...] Elles [les icônes] ont plus à voir avec le caractère vivant de la vérité que les symboles et les indices. [...] Mais il y a une assurance que l'icône apporte au plus haut degré. [...] ce qui est affiché devant le regard de l'esprit - la forme de l'icône est aussi son objet - doit être logiquement possible. (C. S. Peirce, C.P. 4.531. Trad. dans Fisette, 1996: 277.)

On pourrait sans doute entretenir certaines réticences à imaginer que la simple empreinte aperçue par Robinson puisse constituer une illustration, le moindrement convaincante, de cette avancée extrêmement hardie, à savoir que l'icône, parce qu'elle partage avec son objet les mensonges et les déceptions, est au plus proche du caractère vivant de la vérité. Comment, en effet, soutenir que le mensonge et la déception puissent servir de garant à la vérité?

On pourrait imaginer que l'icône puisse être le signe le plus fragile parce qu'il échappe à l'épreuve de la réalité; mais ce serait là une analyse trop restrictive et le texte de Peirce est suffisamment clair pour interdire une telle déperdition de sens: les signesreprésentations que sont les indices et les symboles sont, par définition, des artefacts; et pour cette raison même, ils peuvent être volontairement mensongers, ils peuvent être falsifiés, pis, ils peuvent être figés dans des habitudes sclérosées et désigner des vérités autrefois nécessaires qui, n'ayant plus cours, ne sont plus que des souvenirs encombrants. Bref, ces signes portent court, ils ont perdu leur dynamisme, car ils ne font plus rêver; on pourrait suggérer qu'ils sont trop abstraits, trop exclusivement conventionnalisés pour porter le caractère vivant de la vérité. C'est en cela qu'ils se situent en dehors de la sphère esthétique et c'est précisément pour cette raison qu'à la différence des signes de l'art et en particulier ceux de la musique, ils ne nous apportent pas de satisfaction émotionnelle et qu'ils sont impuissants à constituer le centre d'un rayonnement où l'esprit trouverait une "compréhension musicale» (musical understanding), c'est-à-dire une assurance qui soit d'une nature autre que purement formelle puisque l'émotion y remplit une fonction centrale.

En regard des symboles, les icônes sont plus souples, plus fluides, plus présentes à la conscience et aussi plus près de nos rêves; n'étant pas, comme les indices, liées à un objet extérieur auquel elles seraient soumises comme une esclave à son maître, les icônes peuvent porter un matériau sémiotique plus subtil, ce qui a été particulièrement bien mis en évidence par Susanne Langer qui, pour caractériser le signe musical, exclut les termes de l'alternative classique, soit le schéma "opaque" versus "transparent», pour retenir le mot chatoyant qui désigne les caractères instable, mouvant et donc «vivant de la vérité» du signe musical.

Poursuivons la citation de Peirce: «ce qui est affiché au regard de l'esprit doit être logiquement possible»; c'est là ce que fait l'icône et qui définit strictement le virtuel: afficher des possibilités. Le texte de Langer est très proche car, encore ici, la musique répond d'une façon parfaitement juste à cette fonction de l'icône: «rendre les choses concevables plutôt que de les enfermer dans des propositions». L'étonnante coïncidence qui est ici mise en évidence n'est pas le fait du hasard: dans les deux cas de la musique et de l'icône, c'est le caractère d'indétermination, quant au contenu porté, qui s'avère prédominant, ce qui répond parfaitement à la modalité du virtuel.

Devant divers autres signes où un objet extérieur, garantissant la figuration, ressemble à une ombre toujours immanente, on pourrait négliger cette étrange pression exercée par l'indétermination du virtuel; dans l'écoute musicale, le signe semble loger de l'autre côté de la ligne de partage de lumière: il est lui-même l'ombre de quelque chose d'indéfini. C'est la raison 
essentielle pour laquelle la musique serait particulièrement apte à porter l'émotion, car l'émotion est une charge qui, provenant de l'obscur, ne se laisse pas saisir, ni contrôler. L'émotion nait d'une pulsion que l'on pourrait imaginer comme un nœud obstruant l'obscure passerelle entre la conscience et le monde. L'enjeu du mouvement sémiosique c'est alors de négocier avec cette obstruction, de l'absorber, d'en faire sa matière et d'arriver ainsi à libérer le passage et à assurer une des cohérences possibles entre une visée qui surgit de l'intérieur et le réel objectif. On comprendra dès lors que l'émotion ${ }^{10}$ repose sur le dynamisme propre au mouvement de la sémiose; et si la musique - essentiellement fondée sur des enchevêtrements d'écarts et de résolutions, de tensions et de libérations - s'avère un conducteur si efficace de l'émotion, c'est précisément parce que son mouvement, qui est celui-là même de la sémiose, est une avancée - et dans les meilleurs cas, une réalisation - vers cette adéquation entre l'affect et le monde; c'est ainsi que l'on peut conférer un nouvel effet de sens au texte de Chabanon (1785: 19) qui écrivait que «la musique est émouvante parce qu'elle est mouvante».

On peut d'ailleurs inférer que c'est précisément sur la base de cette proposition que l'on arrive aussi à comprendre que la musique agisse simultanément comme signe de la conscience et signe du monde; d'où la métaphore classique, remontant à Pythagore, de la consonance musicale comme signe de l'harmonie du cosmos, et donc comme icône d'une conception logiquement cohérente et d'une perception sensible, émotionnellement satisfaisante, du monde.

L'écart entre cette conception et la théorie dominante en sémiologie musicale est immense: la plupart des théoriciens ont conclu, en effet, que la musique ne constitue pas un signe: la formule lue et entendue plus de mille fois est simpliste: «la musique est faite de signifiants sans signifiés", comme si le «signifié» se confondait avec le référent. La musique n'a pas de référent; ses actes sonores ne sont pas davantage liés à un signifié qui serait une classe fixe de référents. Mais, en revanche, l'acte musical porte une immense réserve d'effets de sens possibles qui sont non limités et non limitables; et dans la mesure où ces effets de sens débordent le contrôle de la raison, ils assurent un lieu privilégié pour l'éveil d'émotions, tout aussi variables, qui seront relatives au sujet, au lieu, voire à l'heure du jour. D'ailleurs, s'il n'en était pas ainsi, pour quelle raison réécouterait-on une même musique? C'est pourquoi, il serait plus juste d'imaginer que cette musique que l'on écoute et réécoute ressemble au fleuve, dont parlait Héraclite, dans lequel on ne se baigne jamais deux fois.

C'est aussi pour cette même raison que l'on ne saurait imaginer la musique comme l'antithèse du signe (linguistique, par exemple); au contraire la musique affiche l'origine, la richesse préalable et la condition de tout signe; on comprendra que ces trois termes pointent tous en direction de l'affect. Ce n'est d'ailleurs pas sans raison que David Savan (1976: 138) construisit sa "Théorie sémiotique de l'émotion" autour de ce fragment qui situe l'origine de toute inférence précisément dans ce lieu où les certitudes sont ébranlées: «La logique ne dépend de rien que du combat pour échapper au doute lequel, comme il s'achève dans l'action, doit débuter dans l'émotion" (C.P. 2.655, trad. Savan: 1976).

\section{ET SI L'ESSENTIEL DE L'ICÔNE, C'ÉTAIT L'ÉMOTION!}

On peut donc maintenant poser crûment la question essentielle en revenant à Douglas Greenlee qui, le premier, lança naguère le débat, à l'adresse des universitaires spécialistes de Peirce, en interrogeant le statut sémiotique d'une sonate pour piano:

In discussing the range of a general theory of signs, I suggested that objects as diverse as words and piano sonatas are signs. Now the everyday use of language is prepared to admit words and linguistic expressions into the range of signs but would he strained to admit most piano sonatas. And does not one of the reasons lies in the fact that words often "stand for" things, whereas most piano sonatas do not? (Greenlee, 1973: 54)

Dans une étude extraordinairement intéressante, Anne Freadman (1994) cherche à apporter une réponse à cette question. Au terme de l'analyse minutieuse des principales pièces de la 
correspondance avec Lady Welby, elle en arrive à une position qui rejoint la mienne: elle inverse la proposition classique en suggérant que le signe musical démontre, par son existence même, que les fonctions de représentation et de figuration ne sont pas essentielles au signe. Puis elle ajoute - avec sagacité - que c'est précisément le délestage de ces notions qui permet de redonner la première place aux deux principes qui sont les apports les plus originaux de Peirce à la sémiotique: la définition du signe comme action et l'idée de la semiosis ad infinitum.

La position d'Anne Freadman est parfaitement cohérente avec la proposition que je défends ici, dans la mesure où l'idée du signe comme action puis celle du prolongement indéfini de cette action (c'est ce que signifie l'expression semiosis ad infinitum) trouvent une correspondance particulièrement convaincante dans le matériau musical. De fait, on doit reconnaître qu'il y a une cohésion entre la prise en compte de ce que Peirce appelait le "caractère vivant de la vérité», qui est lié aux traits spécifiques de la priméité, et la valeur exemplaire de l'acte musical qui, en substituant le régime de l'audible à celui du visible, vient remettre en cause les certitudes trop rapidement arrêtées concernant les fonctions de figuration et de représentation. C'est précisément là ce que je cherchais à tirer au clair lorsque, plus haut, j'ai suggéré l'idée d'une "tache aveugle» dans la semeiotic ${ }^{11}$.

On pourrait même imaginer que la maturation de la pensée sémiotique de Peirce ait correspondu à une telle avancée ${ }^{12}$. En témoigne ce phénomène qui demeure tout de même étonnant: à mesure que l'on avance dans la chronologie des écrits, la musique, comme modèle logique de référence, devient plus présente (même si cette présence reste modeste), la définition du signe est de moins en moins figurative et le mouvement de sémiose s'associe de plus en plus à l'inférence abductive; le texte qui marque de la façon la plus convaincante cet aboutissement est certainement L'Argument négligé ${ }^{13}$, où apparaît cette notion, devenue aujourd'hui incontournable dans toute réflexion qui porte sur la sémiotique dans ses rapports avec les processus de la créativité: le Musement. Le
«Musement», on le sait, est essentiellement un voyagement de l'esprit dans les territoires de l'ombre, là où les icônes et les rêves se confondent; on sait par ailleurs que c'est précisément là que trouve à s'inscrire un rythme marquant les avancées imprévisibles de l'imaginaire, qu'on pourrait se représenter comme «le fil d'une mélodie», pour reprendre cette expression qu'utilisait Peirce ${ }^{14}$ pour désigner l'action de penser. Jankélévitch se référait certainement à une telle compréhension des actes de cognition lorsqu'il écrivait que «la musique [est] l'adverbe de manière de la pensée» ${ }^{15}$. Tel est le mode d'existence de la pensée au niveau très "archaïque» de l'icône et que la métaphore musicale nous permet de nous représenter avec, peuton imaginer, la plus grande justesse.

\section{L'ICÔNE EST UN GAGE}

\section{DE LA LIBERTÉ DE NOTRE IMAGINAIRE}

Si le signe ne trouvait pas sa racine dans ce lieu de l'icône, les complexités du réel seraient déjà exhaustivement représentées et donc contenues dans divers systèmes de signes; d'une certaine façon, tout serait déjà joué d'avance, on tomberait dans le déterminisme le plus primaire, il n'y aurait plus de place pour l'émotion, et le virtuel, plutôt que de garantir la liberté de l'esprit, ne serait qu'un truc de foire ou un jeu d'illusions, ce que vient nier avec force le discours de la sémiotique; c'est pourquoi celle-ci parle essentiellement des avancées de la signification comme de virtualités de sens.

\section{NOTES}

1. Je préfère la forme peircéen à peircien.

2. Je me permets de souligner ici l'étonnante correspondance dans l'acception du mot secondaire de la psychanalyse (élaboration secondaire) et de la sémiotique (la catégorie de la secondéité).

3. La série "similarité/contiguïté/conventionnalité» ne peut que s'avérer inadéquate à caractériser les catégories phanéroscopiques pour la raison bien simple qu'elle répond à la logique d'un paradigme et non d'une triade.

4. À ce propos, on pourra se référer aux chapitres 8 et 9 de Fisette, 1996. 5. À l'exception de quelques passages extrêmement intéressants, tel celui (C.P. 8.183. Fisette, 1996: 270) où Peirce écrit du mot «soleil» qu'il est une icône sonore de l'objet soleil. 
6. Un phénoménologue et musicologue, F. Joseph Smith, a posé précisément cette question en soulignant que la phénoménologie repose traditionnellement sur une perception visuelle (fondant le terme même de phénoménologie), alors que l'on pourrait envisager un élargissement des perspectives en adjoignant une prise en compte de la perception auditive: "According to Smith (F. Joseph Smith, phenomenologist and musicologist), emphasis on the visual dimension of experience has worked to the detriment of a phenomenology of sound and music, and he acordingly calls for phenomenology to be enriched by an "akoumenology" ". Cité dans Coll., 1997: 469.

7. Alors qu'à l'inverse, les nombreux témoins sonores qui scandent notre vie quotidienne (sonneries, sirènes, thèmes musicaux, etc.) agissent précisément comme indices et, dans de tels cas, la fonction iconique, qui, suivant les règles de la hiérarchie, est toujours présupposée, est reléguée au second plan.

8. Dans ce chapitre VIII, consacré à la musique, de l'ouvrage de Langer on ne trouve aucune référence à l'œuvre de Peirce. Par contre, dans un des premiers chapitres, elle signale les Collected Papers, désignant le modèle de la sémiotique qui s'y élabore comme un ensemble trop complexe pour s'avérer vraiment utile. C'est d'ailleurs là une perception de l'œuvre de Peirce qui prévalait à l'époque, dans les années quarante, et que, assez étonnamment, l'on rencontre encore aujourd'hui. Lors de son arrivée aux États-Unis durant ces mêmes années, Roman Jakobson fut le premier à attirer l'attention des sémioticiens sur l'œuvre fondatrice de Peirce.

9. À propos de cette question, on pourra se référer au vol. $25, n^{\circ} 2$ de la revue Protée (1997): "Musique et procès de sens ». On trouvera aussi au chapitre V ("Le symbolisme musical») de Nattiez (1987) une présentation exhaustive de cette problématique.

10. Je m'appuie ici sur des conceptions étonnamment semblables de l'émotion que je trouve autant chez le jeune Peirce que chez Igor Stravinsky.

"Dans l'inférence hypothétique, la sensation d'une complexité est remplacée par un sentiment simple, mais d'une grande intensité, appartenant à l'acte d'avancer une conclusion hypothétique. Lorsque notre système nerveux est excité par une complication, il s'établirait une relation entre les constituants de cette excitation résultant en une perturbation unique que je nomme une émotion. Ainsi les différents sons produits par les instruments de l'orchestre frappent notre oreille et le résultat en est une émotion particulière, somme toute différente des sons eux-mêmes». (C.P. 2.643, trad. Fisette)

"Le phénomène de la musique nous est donné à la seule fin d'instituer un ordre dans les choses y compris et surtout un ordre entre l'homme et le temps. Pour être réalisé, il exige donc nécessairement et uniquement une construction. La construction faite, l'ordre atteint, tout est dit. Il serait vain d'y chercher ou d'en attendre autre chose. C'est précisément cette construction, cet ordre atteint qui produit en nous une émotion d'un caractère tout à fait spécial, qui n'a rien de commun avec nos sensations courantes et nos réactions dues à des impressions de la vie quotidienne». (Stravinsky, 1935: 63-64. Les caractères italiques sont de l'auteur.)

11. C'est intentionnellement que j'ai inscrit dans cette phrase deux métaphores ("tirer au clair"; " tache aveugle») liées au régime visuel. Par contre, il semblerait que ces difficultés ne deviennent perceptibles qu'à la condition que le sujet qui pose cette interrogation se situe lui-même dans le registre de l'audible. Ce qui expliquerait l'apport extrêmement précieux que représente le texte de S. Langer dans cette discussion sur l'icône.

12. On trouvera une analyse faite sur la base de cette hypothèse dans Fisette, 1996a.
13. "Un argument négligé en faveur de l'existence de Dieu " (C.P. 6.452-485). Ce texte est daté de 1908 et c'est le dernier texte publié de Peirce. On trouvera une traduction en français dans Deledalle, 1990: 172-192.

14. Dès 1878 , dans «Comment rendre nos idées claires? ", Peirce écrivait: "La pensée est comme le fil d'une mélodie qui parcourt la suite de nos sensations." ( C.P. 5.395-397).

15. "On ne pense pas "la musique", mais par contre on peut penser selon la musique, ou en musique, ou musicalement, la musique étant l'adverbe de manière de la pensée» (1961: 127).

\section{RÉFÉRENCES BIBLIOGRAPHIQUES}

ChabANON, M.-P. G. de [1785]: De la musique considérée en elle-même et dans ses rapports avec la parole, les langues, la poésie et le théâtre, Genève, Slatkine Reprints, 1969, 459 p.

COLL. [1997]: Encyclopedia of Phenomenology, Boston, Kluwer Academic Publishers.

Deledalle, G. [1990] : Lire Peirce aujourd'hui, Bruxelles, De Boeck, 217 p. FISETTE, J. [1996]: Pour une pragmatique de la signification. Suivi d'un choix de textes de Charles S. Peirce en langue française, Montréal, XYZ éditeur, coll. «Documents", 300 p.;

[1996a]: «De l'imaginaire au musement. Quelques occurrences de la métaphore musicale dans le texte de C.S. Peirce", dans Texte 17-18, Toronto, 33-57.;

[1997]: "Faire parler la musique. À propos de "Tous les matins du monde" ", dans Protée, vol. 25, n² , automne, 85-97.

FrEADMAN, A. [1994]: "Music “in” Peirce", dans Versus : Quaderni di studi semiotici 64 , 75-95

GenetTe, G. [1976] : Mimologiques, Paris, Seuil.

Greenlee, D. [1973]: Peirce's Concept of Sign, The Hague, Mouton, coll. "Approaches to semiotics" 5, $148 \mathrm{p}$.

HANSLICK, É. [1854| : Du beau dans la musique. Essai de réforme de

l'esthétique musicale ("Introduction" par J.-J. Nattiez), Paris, Christian Bourgois, 1986, coll. "Musique/Passé/Présent», 177 p.

JANKÉLÉVITCH , V. [1961] : La Musique et l'Ineffable, Paris, Seuil, 1983, $194 \mathrm{p}$.

LANGER , S. K. [1942]: "On Significance in Music», dans Philosophy in a New Key. A Study in the Symbolism of Reason, Rite, and Art, Cambridge, Mass., Harvard University Press, 1967, 205-245.

LÉVI-STraUss, C. [1962]: Le Totémisme aujourd'hui, Paris, P.U.F., 1980, 160 p.

NATTIEZ, J.-J. [1987] : Musicologie générale et sémiologie, Paris, Christian Bourgois, $401 \mathrm{p}$.

PAQUIN, N. [1996] : «Percevons-nous vraiment des signes?», dans Visio, 3, 77-84.

PEIRCE, C.S. [1931-1935]: The Collected Papers (C.P.), vol. I-VI (sous la dir. de C. Hartshorne et P. Weiss), Harvard, Harvard University Press;

[1958] : The Collected Papers, vol. VII-VIII (sous la dir. de W. Burks), Harvard, Harvard University Press.

Rousseau, J.-J. [1781] : Essai sur l'origine des langues. Préface et commentaires de J.-L. Schefer, Paris, Presses-Pocket, coll. «Agora Les classiques » 60, 1990, 248 p.

SAVAN, D. [1976] : "La théorie sémiotique de l'émotion selon Peirce» (trad. de "Peirce's Theory of Emotion"), dans Nouvelle Revue d'Ethnopsychiatrie 11, 1988, 127-146.

STRAVINSKY, I. [1935] : Chroniques de ma vie, Paris, Denoël-Gonthier, coll. «Médiations » 83, 1971, 63-64.

Tiercelin, C. [1993]: La Pensée-signe. Études sur C. S. Peirce, Nîmes, Éd. J. Chambon, coll. "Rayon Philo», 399 p. 